\title{
Chalcogenide Glass Fibers: Optical Window Tailoring and Suitability for Bio-Chemical Sensing
}

\author{
Pierre Lucas ${ }^{1} *$, Garret J. Coleman ${ }^{1}$, Shibin Jiang ${ }^{2}$, Tao Luo ${ }^{2}$, Zhiyong Yang ${ }^{3}$ \\ ${ }^{1}$ Department of Materials Science and Engineering, University of Arizona, Tucson, Arizona 85712 \\ ${ }^{2}$ AdValue Photonics, 3440 E Britannia Dr \#190, Tucson, AZ 85706, USA \\ ${ }^{3}$ Jiangsu Key Laboratory of Advanced Laser Materials and Devices, School of Physics and Electronic \\ Engineering, Jiangsu Normal University, Xuzhou, Jiangsu 221116, China \\ *Corresponding author:Pierre@u.arizona.edu
}

\begin{abstract}
:
Glassy materials based on chalcogen elements are becoming increasingly prominent in the development of advanced infrared sensors. In particular, infrared fibers constitute a desirable sensing platform due to their high sensitivity and versatile remote collection capabilities for insitu detection. Tailoring the transparency window of an optical material to the vibrational signature of a target molecule is important for the design of infrared sensor, and particularly for fiber evanescent wave spectroscopy. Here we review the basic principles and recent developments in the fabrication of chalcogenide glass infrared fibers for application as biochemical sensors. We emphasize the challenges in designing materials that combine good rheological properties with chemical stability and sufficiently wide optical windows for biochemical sensing. The limitation in optical transparency due to higher order overtones of the amorphous network vibrations is established for this family of glasses. It is shown that glasses with wide optical window suffer from higher order overtone absorptions. Compositional engineering with heavy elements such as iodine is shown to widen the optical window but at the cost of lower chemical stability. The optical attenuations of various families of chalcogenide glass fibers are presented and weighed for their applications as chemical sensors. It is then shown that long-wave infrared fibers can be designed to optimize the collection of selective signal from bio-molecules such as cells and tissues. Issues of toxicity and mechanical resistance in the context of bio-sensing are also discussed.
\end{abstract}

Keywords: Infrared Fibers, Fiber Sensors, Amorphous Materials, Far Infrared, Optical Sensors, Infrared Spectroscopy, Evanescent Wave Spectroscopy.

\section{Introduction:}

From industrial window glass to advanced telecom waveguides, amorphous solids are used in a wide range of technological applications thanks to their excellent optical transparency. This desirable property stems from their homogeneous microstructure which can be retained during elaborate fabrication processes such as blowing, drawing or molding. The unique rheological properties of glasses indeed enable the production of complex objects with superior optical quality at minimal cost. This is in contrast to crystalline solids which typically form through 
nucleation and growth and result in materials containing many grain boundaries causing strong light scattering and eventually leading to opacity. This can be overcome by producing single crystals with excellent optical quality, but their synthesis and subsequent shaping is prohibitively expensive for large scale applications. This is the reason why the large majority of optical elements are made of glass, in particular those designed to operate in the visible and nearinfrared regime. In this range of wavelength, silicate glasses vastly dominate the optical elements market due to their superior optical transparency, their excellent glass forming ability, and high chemical durability.

In contrast, mid-infrared systems still largely rely on optical elements made of crystalline solids such as $\mathrm{KBr}, \mathrm{ZnSe}$ or Ge. In particular, Ge has been the material of choice for the fabrication of advanced lenses for night vision systems such as thermal cameras. However, these lenses must be produced from large single crystals subsequently polished by diamond turning and consequently constitute one of the main source of the exceedingly high price of such devices. Nevertheless, there has recently been a shift away from crystalline infrared optics, driven by the development of novel infrared transparent glasses based on chalcogen elements. Indeed, these glasses can be easily molded with high precision to yield aspheric or diffractive lenses and are now produced industrially for a fraction of the price of that of crystalline optics [1-3]. Lenses made of chalcogenide glasses are now commercialized under different brand names and have helped reduce the price of infrared optical system. In particular, night vision cameras equipped with these lenses are now mounted in car bumpers and serve as driving assistance in hazardous conditions such as foggy weather or at night [1]. This type of large scale production is expected to further reduce the price of infrared optics.

But besides thermal imaging, the mid-infrared domain is of great interest for vibrational spectroscopy of chemicals and biomolecules [4-9]. Indeed, almost all molecules exhibit highly specific vibrational signatures in the 2-16 microns range which provides an effective mean of performing selective optical sensing. In particular, biomolecules and microorganisms have distinct mid-infrared signatures which can be used to identify strains or monitor metabolic processes [10-14]. Due to their broad infrared transparency, chalcogenide glasses have therefore been used to fabricate many optical elements for the design of novel infrared biosensors [15-18]. And due to their excellent rheological properties, chalcogenide glasses can be drawn into fibers for the design of remote infrared sensors [19-22]. In this paper we first investigate the source of the fundamental limitation of the optical window in a series of chalcogenide glasses and the corresponding optical fibers, and we present recent development in the production of long-wave infrared fibers suited for the study and monitoring of biomolecules as well as the trade-off between optical properties and chemical stability. 


\section{Experimental:}

\section{Glass synthesis:}

The chalcogenide glasses produced in this study are composed of mixtures of $\mathrm{Se}, \mathrm{Te}, \mathrm{Ge}, \mathrm{As}$ and I. Elements of high purity ( $>99.9999 \%)$ are introduced in a silica tube under high vacuum $\left(\sim 10^{-5}\right.$ Torr) and pumped for several hours to remove all trace of oxygen and water. Surface oxide on Se and As are eliminated by heating the elements in-situ to remove the high vapor pressure oxide species. A small amount of $\mathrm{Al}(50-100 \mathrm{ppm})$ is also added to the mixture to capture remaining oxygen by forming $\mathrm{Al}_{2} \mathrm{O}_{3}$. The resulting mix is then sealed in the silica ampoule under vacuum and introduced in a rocking oven for homogenization at $800^{\circ} \mathrm{C}$ for $12 \mathrm{~h}$. The liquid is then quenched in water to produce a glass rod which is then introduced in a distillation tube. The glass mixture is then distilled in-situ to eliminate the low vapor pressure impurities such as carbon and the $\mathrm{Al}_{2} \mathrm{O}_{3}$ that has formed during the heating process. After distillation the glass is further homogenized at $800^{\circ} \mathrm{C}$ for $6 \mathrm{~h}$, then quenched in water and immediately introduced in an annealing oven set at $\sim 5^{\circ} \mathrm{C}$ below the glass transition temperature $T_{g}$ to remove mechanical constraints and avoid cracking. The temperature is then slowly decreased to room temperature and the glass rod is recovered for processing. Further detail on the purification process can be found in ref [23].

\section{Fiber preparation:}

Glass fibers are produced from purified glass rods by melting the tip of the rod in a narrow ring heater held at a temperature above $T_{g}$. The fiber is then rolled on a Teflon coated wheel and the fiber diameter is monitored in-situ during the drawing process using an optical sensor. The drawing process is performed under He flow to avoid oxidation. The fiber diameter is controlled by adjusting the fiber tension, drawing speed and rate of preform introduction into the heater.

\section{Characterization:}

Optical transmission of glassy samples is measured on discs with thickness of $2 \mathrm{~mm}$ using a FTIR spectrometer Brucker Tensor 27 equipped with an internal DTGS (Triglycerine sulfate) detector. The fiber attenuation is measured using the FTIR spectrometer and a fiber testing attachment equipped with concave gold mirrors for light coupling in and out of the fibers and an external MCT (Mercury Cadnium Telluride) detector cooled with liquid nitrogen. The attenuation is measured using the cut-back method.

\section{Limitation of the Optical Window in Chalcogenide Glasses:}

The optical transmission of amorphous solids is limited on the short wavelength side by electronic absorption processes (mainly controlled by the band gap energy), and on the long wavelength side by vibrational transitions which are mainly controlled by the reduced mass $m^{*}$ and the force constant $k$ of the network oscillators following equation (1) and (2):

$$
E_{n}=\left(n+\frac{1}{2}\right) h v
$$




$$
v=\frac{1}{2 \pi} \sqrt{\frac{k}{m^{*}}}
$$

where $E_{n}$ is the quantized energy of a vibrational state and $v$ is the vibration frequency of the oscillator. While equation (2) is strictly defined for a diatomic oscillator, it provides a good indication of the vibrational properties of a network. Indeed, the extent of the optical window at long wavelengths is a strong function of the mass of component elements as depicted on Fig. 1 for a series of stoichiometric $\mathrm{As}_{2} \mathrm{X}_{3}$ glasses based on arsenic combined with increasingly heavier chalcogenide elements.

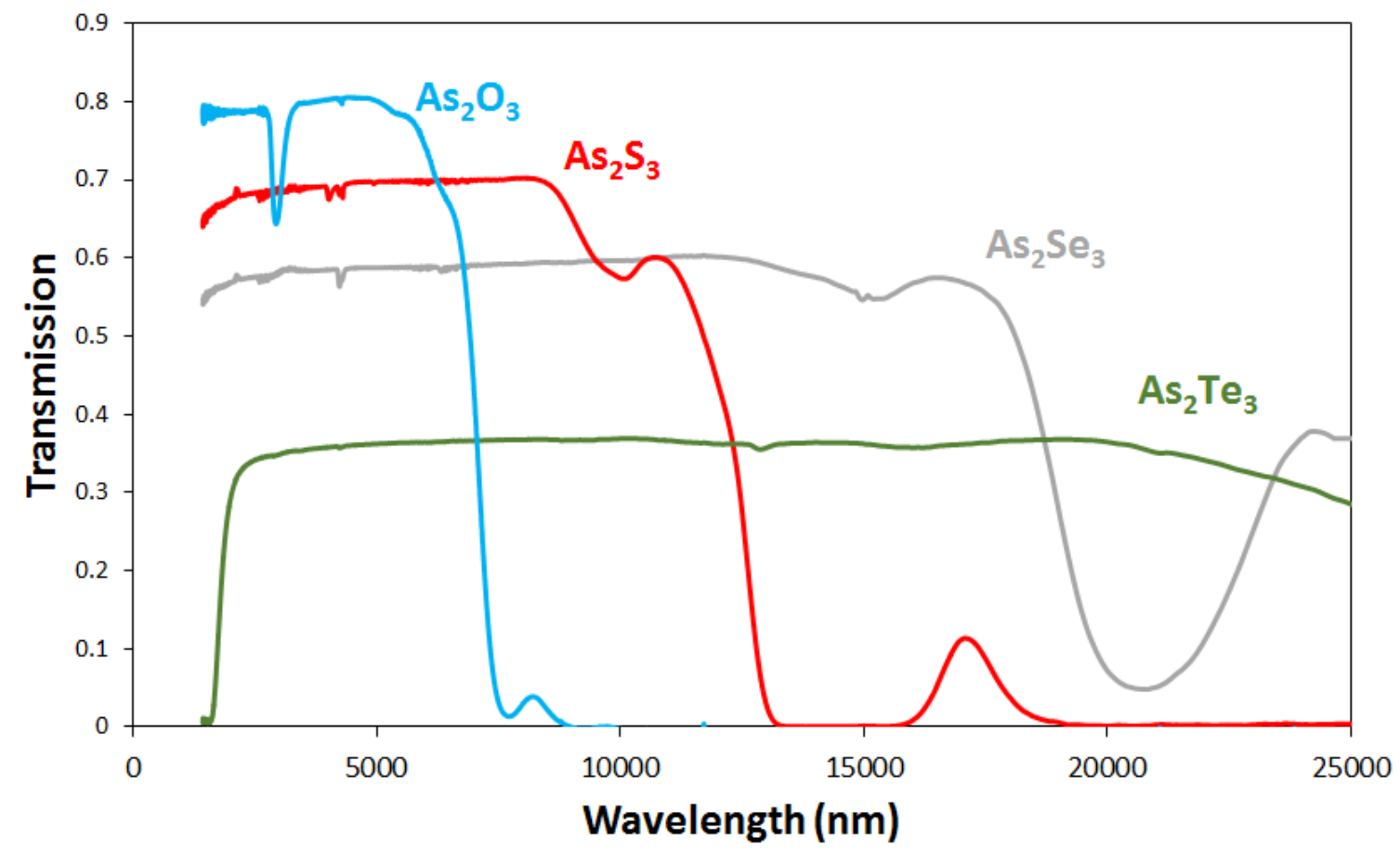

Fig. 1: Long-wave transmission window of amorphous solids composed of increasingly heavier elements. Heavier component elements result in extended transmission in the infrared region. The transmission was measured on 1-2mm thick discs.

Amorphous solids composed of lighter elements such as $\mathrm{As}_{2} \mathrm{O}_{3}$ exhibit strong absorption at relatively high frequency resulting in a multiphonon cut-off at short wavelength near 6-7 $\mu \mathrm{m}$ (Fig. 1). This glass would therefore be inadequate for mid-infrared applications. In addition, $\mathrm{As}_{2} \mathrm{O}_{3}$ is highly hygroscopic as shown by the $\mathrm{OH}$ absorption peak near $3 \mu \mathrm{m}$ (Fig. 1) and as a consequence it degrades and becomes opaque within a few days in ambient atmosphere. Substitution of $\mathrm{O}$ by $\mathrm{S}$ to produce $\mathrm{As}_{2} \mathrm{~S}_{3}$ results in better chemical stability and wider transparency up to $\sim 10 \mu \mathrm{m}$ which is practical for many infrared applications. Indeed $\mathrm{As}_{2} \mathrm{~S}_{3}$ was one of the first glass investigated for infrared applications [24]. Finally, substitution of S by 
increasingly heavier chalcogens Se and Te pushes the multiphonon cut-off far into the infrared down to 18 and $25 \mu \mathrm{m}$ respectively.

The term "multiphonon" cut-off originates from the fact that the absorption edge is limited by the broad phonon density of state characteristic of amorphous structures. But it should also be noted that higher overtones of the fundamental vibrational energy play an essential role in defining the optical window. Vibrational selection rules for a harmonic oscillator do not allow overtone absorptions and only transition involving $\Delta n= \pm 1$ are permissible, but it is clear from Fig. 1 that overtones of multiple orders can induce strong absorptions in these glasses. Indeed the structure of $\mathrm{As}_{2} \mathrm{X}_{3}$ glasses is composed of corner sharing $\mathrm{As} \mathrm{X}_{3}$ trigonal pyramids and the main vibrational mode of this structure is the $A_{1}$ breathing mode depicted in the inset of Fig. 2 . This mode is both IR and Raman active and its fundamental energy can therefore be obtained by Raman spectroscopy as shown in Fig. 2.

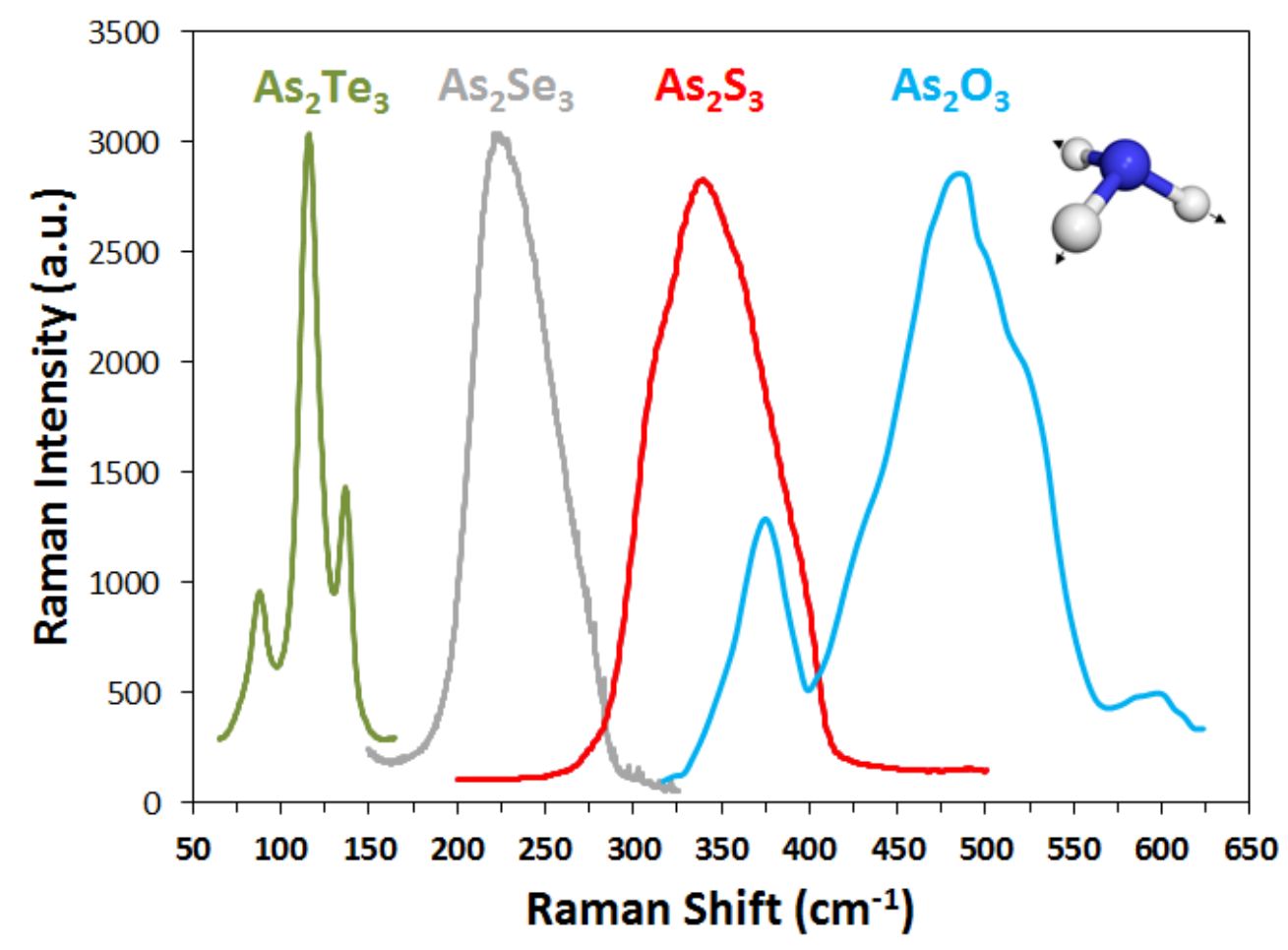

Fig. 2: Raman spectra of $\mathrm{As}_{2} \mathrm{O}_{3}, \mathrm{As}_{2} \mathrm{~S}_{3}, \mathrm{As}_{2} \mathrm{Se}_{3}$ and $\mathrm{As} \mathrm{s}_{2} \mathrm{Te}_{3}$ showing the fundamental contribution of the pyramidal As- $X_{3}$ symmetric stretching modes centered at $485 \mathrm{~cm}^{-1}, 340 \mathrm{~cm}^{-1}, 230 \mathrm{~cm}^{-1}$ and $115 \mathrm{~cm}^{-1}$ respectively.

The absorption wavelengths corresponding to the fundamental vibrational modes and their overtones are listed in Table 1 for each glass. For example the fundamental vibrational energy of $\mathrm{As}_{2} \mathrm{~S}_{3}$ is centered at $340 \mathrm{~cm}^{-1}$, hence it second and third overtones occur at $680 \mathrm{~cm}^{-1}$ and 1020 $\mathrm{cm}^{-1}$ respectively. These three modes correspond to absorption wavelengths at $30 \mu \mathrm{m}\left(340 \mathrm{~cm}^{-1}\right)$, $14.5 \mu \mathrm{m}\left(680 \mathrm{~cm}^{-1}\right)$ and $9.8 \mu \mathrm{m}\left(1020 \mathrm{~cm}^{-1}\right)$ and their relative effect on the optical window can 
be clearly observed in the absorption spectrum of Fig. 1. The fundamental absorption is extremely broad and intense while the second overtone near $14.5 \mu \mathrm{m}$ is less intense but still saturates the absorption. Finally the third overtone at $9.8 \mu \mathrm{m}$ shows a weak absorption through a disc but will prove to be the limiting absorption mechanism through a fiber. A similar observation can be made for $\mathrm{As}_{2} \mathrm{O}_{3}$ and $\mathrm{As}_{2} \mathrm{Se}_{3}$ with the second overtone at $6.9 \mu \mathrm{m}$ and $14.5 \mu \mathrm{m}$ respectively. For $\mathrm{As}_{2} \mathrm{Te}_{3}$ the main absorption band initiating near $21.7 \mu \mathrm{m}$ is actually due to the third overtone. This suggests that the oscillator anharmonicity becomes large for these heavy glasses and allows higher order overtones. This is consistent with the low bond energy for these materials [25] which should result in shallow and distorted Morse potentials.

Table 1: Frequency and wavelength of the fundamental vibrational modes and their overtones for $\mathrm{As}_{2} \mathrm{O}_{3}, \mathrm{As}_{2} \mathrm{~S}_{3}, \mathrm{As}_{2} \mathrm{Se}_{3}$ and $\mathrm{As}_{2} \mathrm{Te}_{3}$ glasses. Calculations of the overtones are based on the approximation of a harmonic oscillator.

\begin{tabular}{lcccccccc}
\hline & \multicolumn{2}{c}{$\mathbf{A s}_{\mathbf{2}} \mathbf{O}_{\mathbf{3}}$} & \multicolumn{2}{c}{$\mathbf{A s}_{\mathbf{2}} \mathbf{S}_{\mathbf{3}}$} & \multicolumn{2}{c}{$\mathbf{A s}_{\mathbf{2}} \mathbf{S e}_{\mathbf{3}}$} & \multicolumn{2}{c}{$\mathbf{A s}_{\mathbf{2}} \mathbf{T e}_{\mathbf{3}}$} \\
\cline { 2 - 9 } & $\mathrm{cm}^{-1}$ & $\mu \mathrm{m}$ & $\mathrm{cm}^{-1}$ & $\mu \mathrm{m}$ & $\mathrm{cm}^{-1}$ & $\mu \mathrm{m}$ & $\mathrm{cm}^{-1}$ & $\mu \mathrm{m}$ \\
Fundamental & 485 & 20.6 & 340 & 29.4 & 230 & 43.5 & 115 & 87 \\
$\mathbf{1}^{\text {rst }}$ overtone & 970 & 10.3 & 680 & 14.7 & 460 & 21.7 & 230 & 43.5 \\
$\mathbf{2}^{\text {nd }}$ overtone & 1455 & 6.9 & 1020 & 9.8 & 690 & 14.5 & 345 & 29 \\
$\mathbf{3}^{\text {rd }}$ overtone & -- & -- & -- & -- & -- & -- & 460 & 21.7 \\
\hline
\end{tabular}

Another feature of importance in Fig. 1 is the decrease in maximum transmission with increasing atomic mass observed for each sample. This feature directly derives from the increasing refractive index resulting from large polarizable elements which induces high reflection losses. Indeed the refractive index of telluride glasses can be as high as 3.5 and result in large losses. This is a critical problem for the design of infrared systems which must be remedied with antireflection coatings or surface patterning [26].

Finally, it should be noted that while the atomic mass affects the IR cut-off, it also controls the short wavelength absorption edge. Indeed, smaller atoms tend to lead to stronger bond energies which are associated with greater stabilization of molecular orbitals and wider band gaps. This pattern is illustrated visually in Fig. 3 which shows the evolution in transparency with decreasing band gap energy $E_{g}$ for $\mathrm{As}_{2} \mathrm{O}_{3} E_{g}=6 \mathrm{eV}, \mathrm{As}_{2} \mathrm{~S}_{3} E_{g}=2.4 \mathrm{eV}, \mathrm{As}_{2} \mathrm{Se}_{3} E_{g}=1.53 \mathrm{eV}, \mathrm{As}_{2} \mathrm{Te}_{3} E_{g}=$ $0.87 \mathrm{eV}$. While most oxides are transparent in the visible, sulfides are usually red while selenide and tellurides are opaque except when they contain significant amounts of alkali halide [27, 28]. 


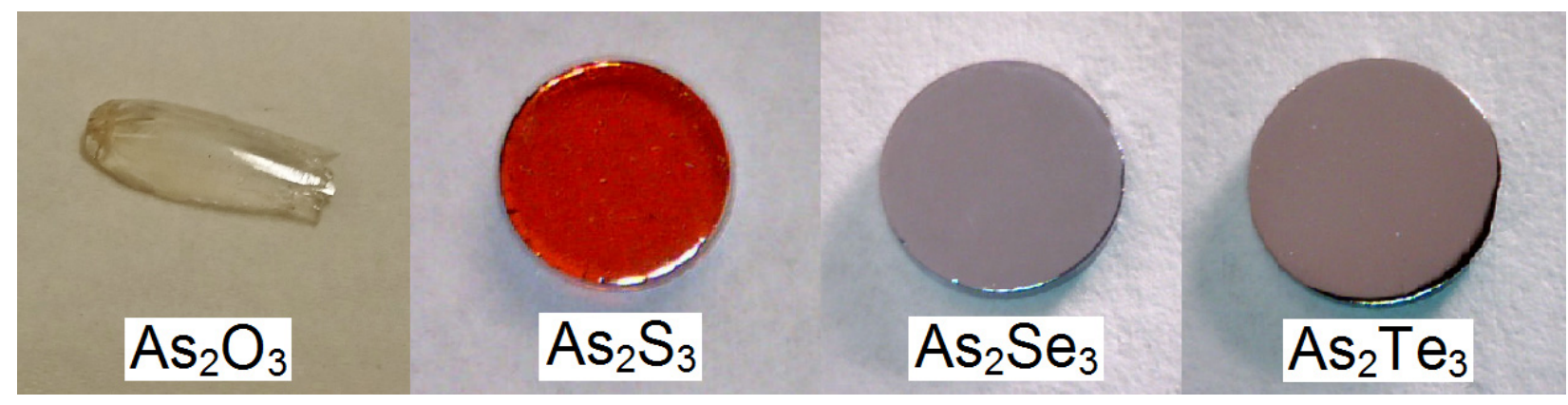

Fig. 3: Glass samples corresponding to the transmission curves shown in Fig. 1. The visible transparency decreases with increasing atomic mass and decreasing band gap energy.

\section{Tailoring the Optical Window of Chalcogenide Glass Fibers:}

Applications such as fiber sensors, fiber imaging bundles or fiber laser delivery involve a long optical path through the fiber which results in strong losses even for weak absorption mechanisms. Fig. 4 illustrates the pronounced contribution of small absorption mechanisms by comparing the losses of a $2 \mathrm{~mm}$ thick disc and an optical fiber made of the same $\mathrm{Ge}_{20} \mathrm{As}_{20} \mathrm{Te}_{45} \mathrm{Se}_{15}$ glass. It is shown that the weak overtone absorption near $12 \mu \mathrm{m}$ results in transmission saturation in the optical fiber. This absorption shoulder is associated with $-\mathrm{Se}$ vibrations and is very weak in the disc but leads to the optical cut-off in the fiber.

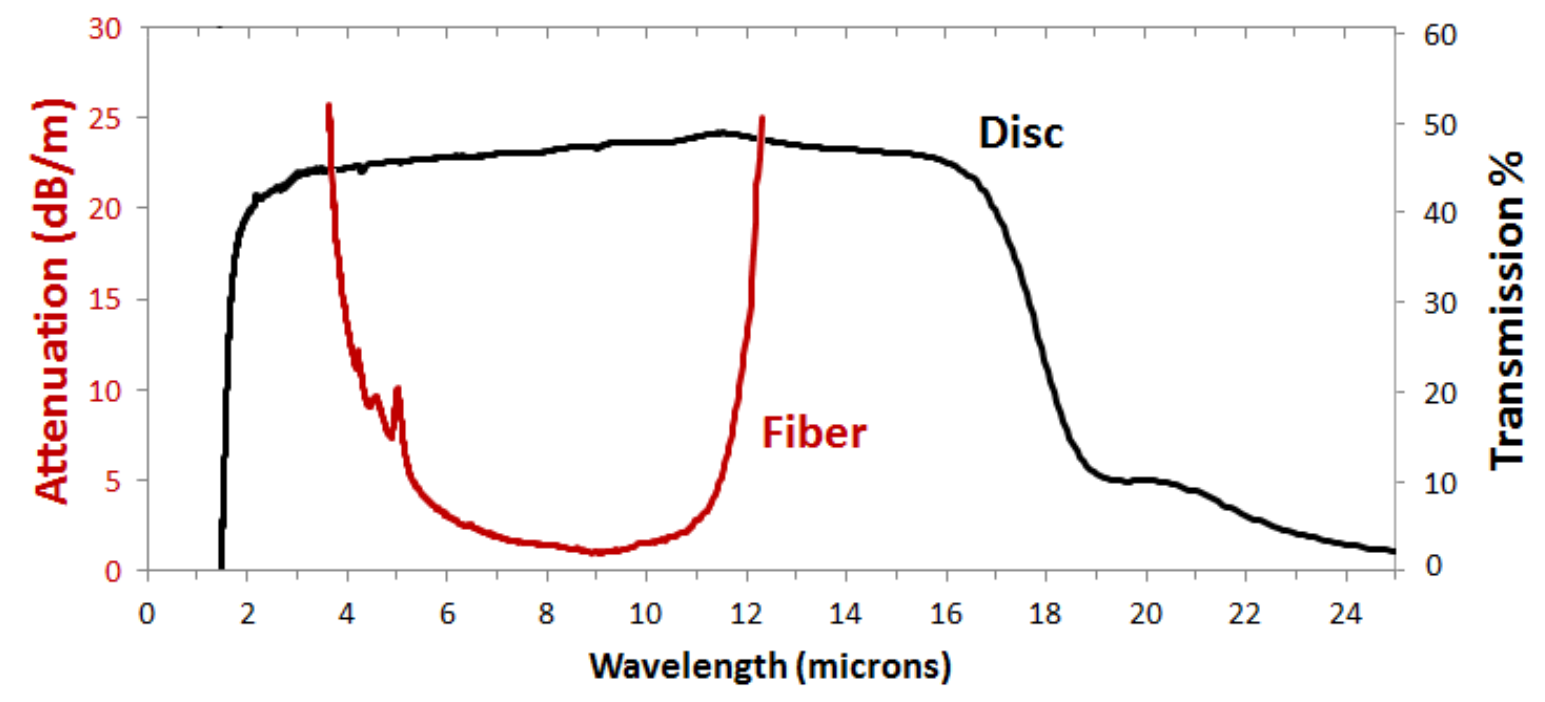

Fig. 4: Comparison of the transmission domain of a $1 \mathrm{~mm}$ thick disc (black) and a fiber (red) made of the same $\mathrm{Ge}_{20} \mathrm{As}_{20} \mathrm{Te}_{45} \mathrm{Se}_{15}$ glass.

Many fundamental applications in infrared optics require high transparency in the long-wave IR range around 6-12 $\mu \mathrm{m}$. This includes thermal imaging centered around $10 \mu \mathrm{m}, \mathrm{CO}_{2}$ laser surgery at $10.6 \mu \mathrm{m}$, communication or countermeasure in the second atmospheric window at $8-12 \mu \mathrm{m}$, or biomolecular spectroscopy from 6-10 $\mu \mathrm{m}$. Fortunately, a wide compositional landscape is 
available to produce chalcogenide fibers with broad IR transmission. Figure 5 illustrate the broad compositional range that is available to tune the optical window of chalcogenide glasses in the mid-infrared.

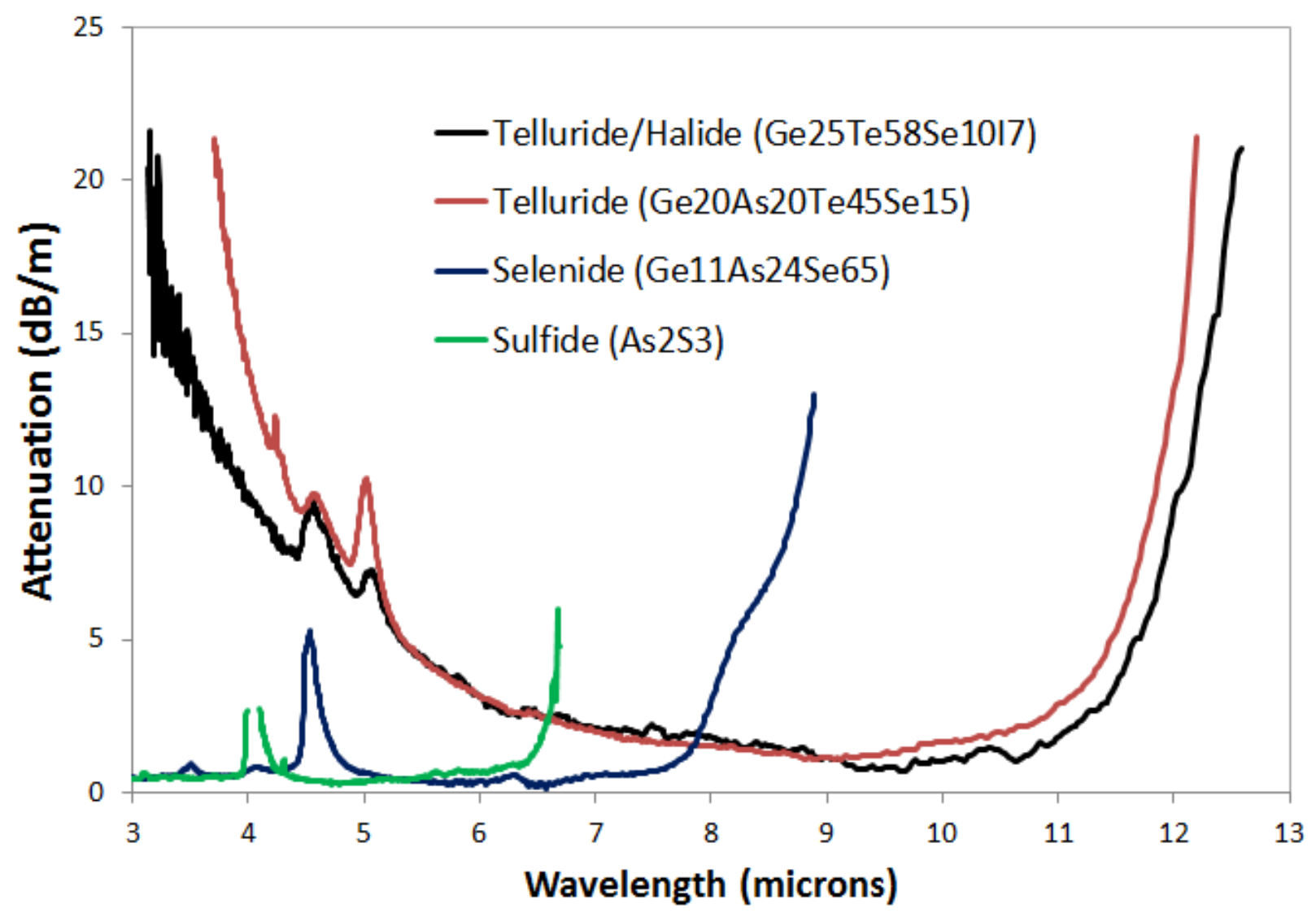

Fig. 5: Comparison of the transmission window of single index fibers composed of $\mathrm{Ge}_{25} \mathrm{Te}_{58} \mathrm{Se}_{10} \mathrm{I}_{7}$ and $\mathrm{Ge}_{20} \mathrm{As}_{20} \mathrm{Te}_{45} \mathrm{Se}_{15}$ glass. The glass containing iodine exhibits a wider window.

The $\mathrm{As}_{2} \mathrm{~S}_{3}$ glass fiber cuts-off near $7 \mu \mathrm{m}$ due to the onset of the third overtone while the GeAsSe fiber cuts-off near 9 microns. In that respect, Fig. 5 indicates that sulfide and selenide glass fibers may be inadequate for many far-infrared applications. In this case, glasses containing tellurium are required to achieve wide IR transparency [23, 29-34].

One of the main challenges for the fabrication of long wave infrared fibers is to obtain a glass with a high tellurium content which does not crystallize during the fiber drawing process [34] and does not exhibit high charge carrier losses [23]. Combining these properties requires significant compositional and structural engineering [33]. For example, addition of either selenium [34] or iodine [35] to telluride glasses can effectively improve the glass formation ability and stabilize the glass against crystallization during fiber drawing. This way, high quality fibers can be obtained from telluride glasses as depicted in Fig. 6(a). Preforms of small diameter typically near $7 \mathrm{~mm}$ are used to minimize temperature gradients and prevent crystallization 
processes during fiber drawing. Core/clad fibers can also be produced using the rod in tube method (Fig. 6(b)). The clad tube is obtained by mechanical drilling approach and the core rod is produced through thermally drawing the original core glass rod to reduce its diameter to the required size. The complete assembly is then drawn a second time into a core/clad optical fiber. Substitution of Se by Te by only $1 \%$ is sufficient to induce the required index change between the core and clad. Single mode fibers can be obtained this way [34].

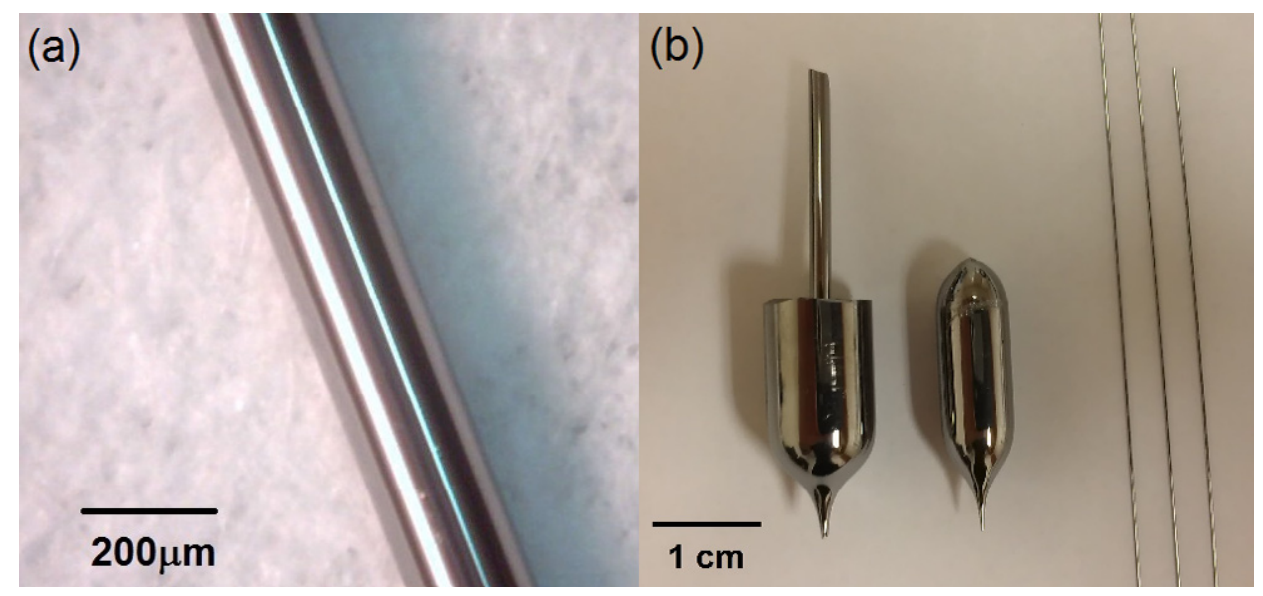

Fig. 6: (a) Ge-As-Te-Se fiber. (b) Preform used to produce single index (right) and core/clad (left) Ge-As-Te-Se fibers.

\section{Optical Properties Versus Chemical Resistance:}

Selenium is notably lighter than iodine which has virtually the same mass as tellurium. As a result, substitution of tellurium by iodine rather than selenium yields optical windows that extend further in the IR as shown in Fig. 5. In addition to its heavy mass, iodine also has a higher electronegativity which raises the ionic character of the bonds and slightly increases the band gap energy. As a result, the short wavelength cut-off is also extended towards high frequency. Hence, the addition of iodine is quite beneficial as it permits to widen the optical window simultaneously on both sides. However, the synthesis of iodine glass is complicated due to its highly volatile nature, especially during the purification steps so that tight control of the stoichiometry can be challenging. But more importantly, glasses containing large quantities of iodine exhibit lower chemical stability over long time exposure to ambient air as depicted in Fig. 7. Two bare fibers of compositions $\mathrm{Ge}_{20} \mathrm{As}_{20} \mathrm{Te}_{45} \mathrm{Se}_{15}$ and $\mathrm{Ge}_{25} \mathrm{Te}_{58} \mathrm{Se}_{10} \mathrm{I}_{7}$ were exposed to air for three years in the dark. Figure 7 shows significant sign of damage on the fiber containing iodine while the GeAsTeSe fiber shows no visible sign of corrosion. In fact the iodine-containing fiber entirely lost its transparency during after that period of time, while the telluride fiber retained its transparency. Indeed, the excellent chemical stability of telluride and selenide glasses has been previously investigated and it was shown that telluride glasses do not show any sign of corrosion even after immersion in water for several days [36], while selenide fiber show negligible corrosion after extended air exposure and do not show measurable loss in transparency [37]. 
Nevertheless the apparent low chemical stability of iodine containing fiber could be greatly alleviated with application of protective coatings which is fairly standard in commercial fiber manufacturing.

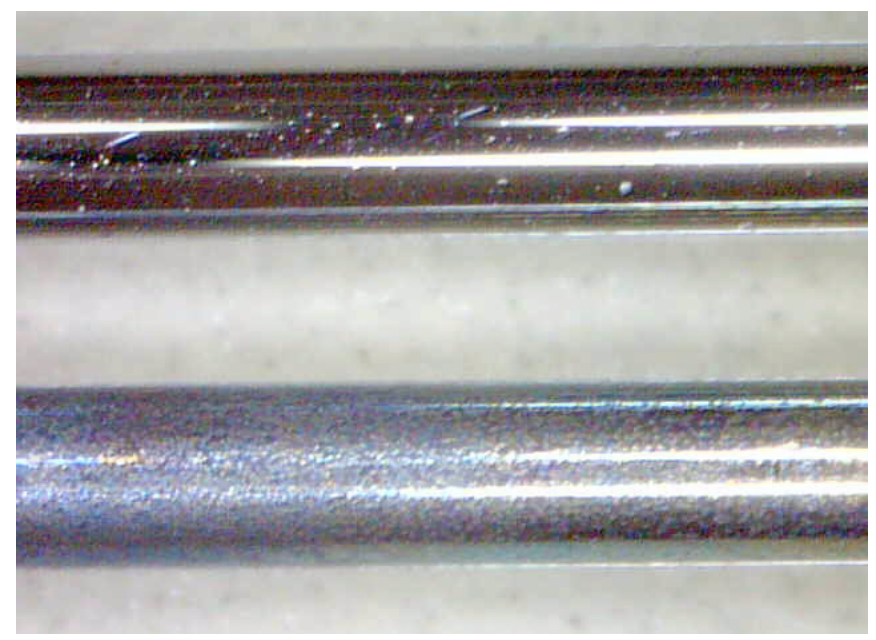

Fig. 7: Microscope image of two fibers of compositions $\mathrm{Ge}_{20} \mathrm{As}_{20} \mathrm{Te}_{45} \mathrm{Se}_{15}$ (top) and $\mathrm{Ge}_{25} \mathrm{Te}_{58} \mathrm{Se}_{10} \mathrm{I}_{7}$ (bottom) exposed to air for 3 years. The fiber containing iodine shows significant surface damage. The fiber diameter is $200 \mu \mathrm{m}$.

\section{Long-Wave Chalcogenide Glass Fibers for Chemical and Biological Sensing:}

\subsection{Optical Window}

As mentioned in the previous section, many infrared applications require optical elements with high transparency in the 6-12 $\mu \mathrm{m}$ region. This is particularly the case of chemical and biological sensing where critical vibrational signal may lay in this wavelength region. As an example, the performance for chemical sensing of a sulfide, selenide and telluride fiber are compared in Fig. 8. The spectra of a standard target molecule (ethylphenol) was collected by fiber evanescent wave spectroscopy (FEWS) using an $\mathrm{As}_{2} \mathrm{Se}_{3}$ fiber, a GeSe fiber, a $\mathrm{Ge}_{15} \mathrm{As}_{40} \mathrm{Te}_{40} \mathrm{Se}_{5}$ fiber and compared with a reference spectra obtained by ATR. The FEWS method is described in detail elsewhere [20,38] but involves coupling an FTIR light source into one end of a tapered fiber in contact with the analyte and collecting the signal with an MCT detector at the other end. The results of Fig. 8 clearly illustrate the superiority of the telluride fiber for collecting a broader range of IR signal, while the selenide and sulfide fibers cut-off significant sections of the molecular signature. However, it should be noted that the optical window of the telluride cuts-off earlier than its counterparts near $3 \mu \mathrm{m}$ due to its low band-gap energy. In that respect, iodine containing fibers would provide a significant advantage by opening the window on both sides of the spectra. 


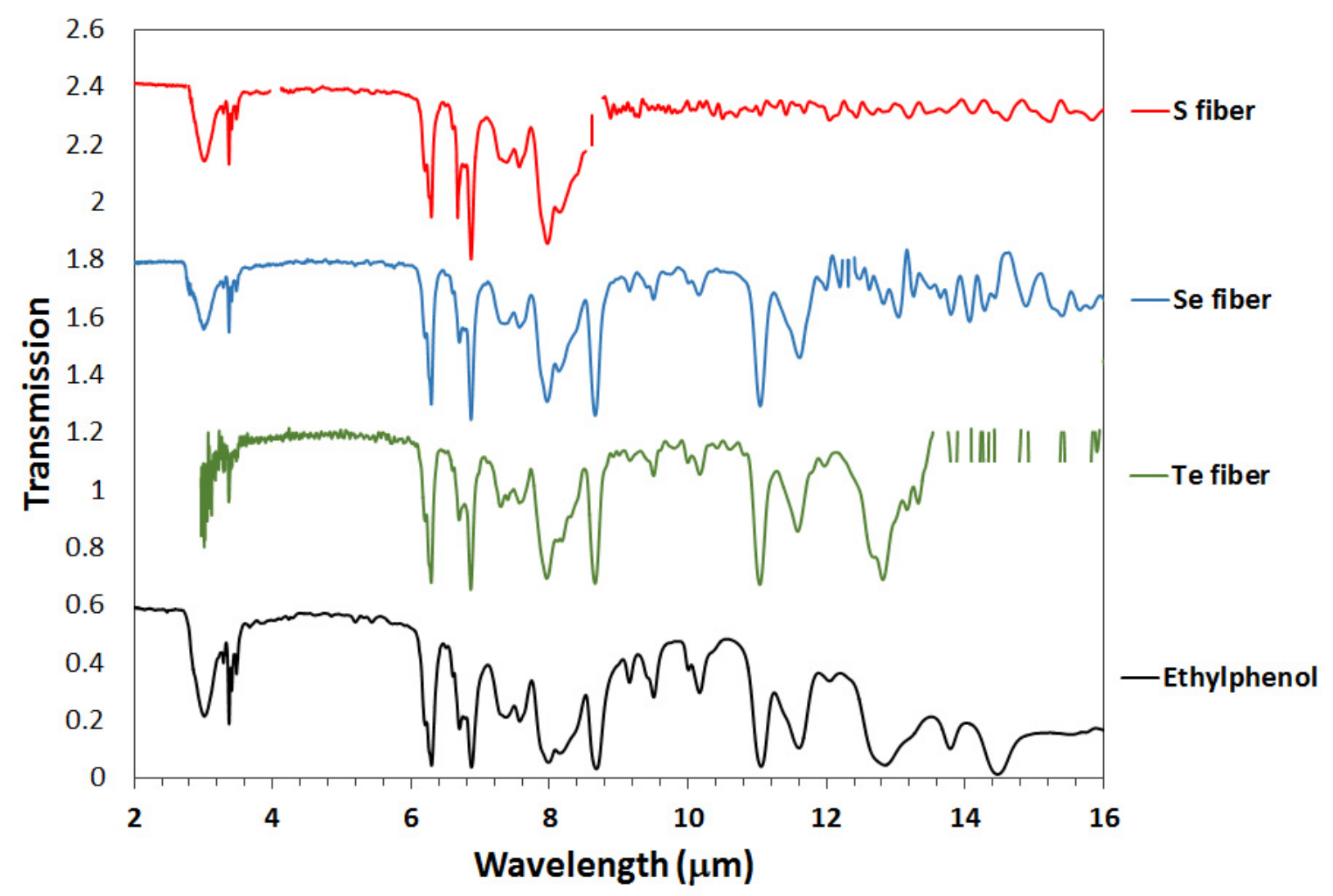

Fig 8: Vibrational spectra of ethylphenol collected by fiber evanescent wave spectroscopy using from top to bottom a $\mathrm{As}_{2} \mathrm{Se}_{3}$ fiber, a GeSe 9 fiber, a $\mathrm{Ge}_{15} \mathrm{As}_{40} \mathrm{Te}_{40} \mathrm{Se}_{5}$ fiber, and compared with a reference spectra obtained by ATR. Singularities in the regions of low transmission were cut-off for clarity.

The ability to collect high quality signal in the $6-12 \mu \mathrm{m}$ region is even more critical for biological sensing. The complex vibrational signature of bio-molecules in the $6-12 \mu \mathrm{m}$ region offers a very effective mean of identifying, monitoring or diagnosing bio-molecules and tissues [10, 11, 3941]. In that respect chalcogenide glass bio-sensors have been successfully used to diagnose cancerous tissues [42] or human serum [43], as well as to monitor the swarming of bacterial film [44] or the metabolism of live lung cell cultures [13] and to selectively identify different types of bacteria [15] or proteins [33].

Infrared optical fibers offer one of the most desirable sensor configuration for this purpose because they can be tapered to enhance sensitivity [20,45] and enable remote or in-situ sensing. In addition, Fig. 7 shows that the optical window of telluride fibers can be tailored to match the highly selective "signature" region of biomolecules where the signal of each cell component is located, including amides I and II, phospholipids, nucleic acids, fatty acids and amino acids. 


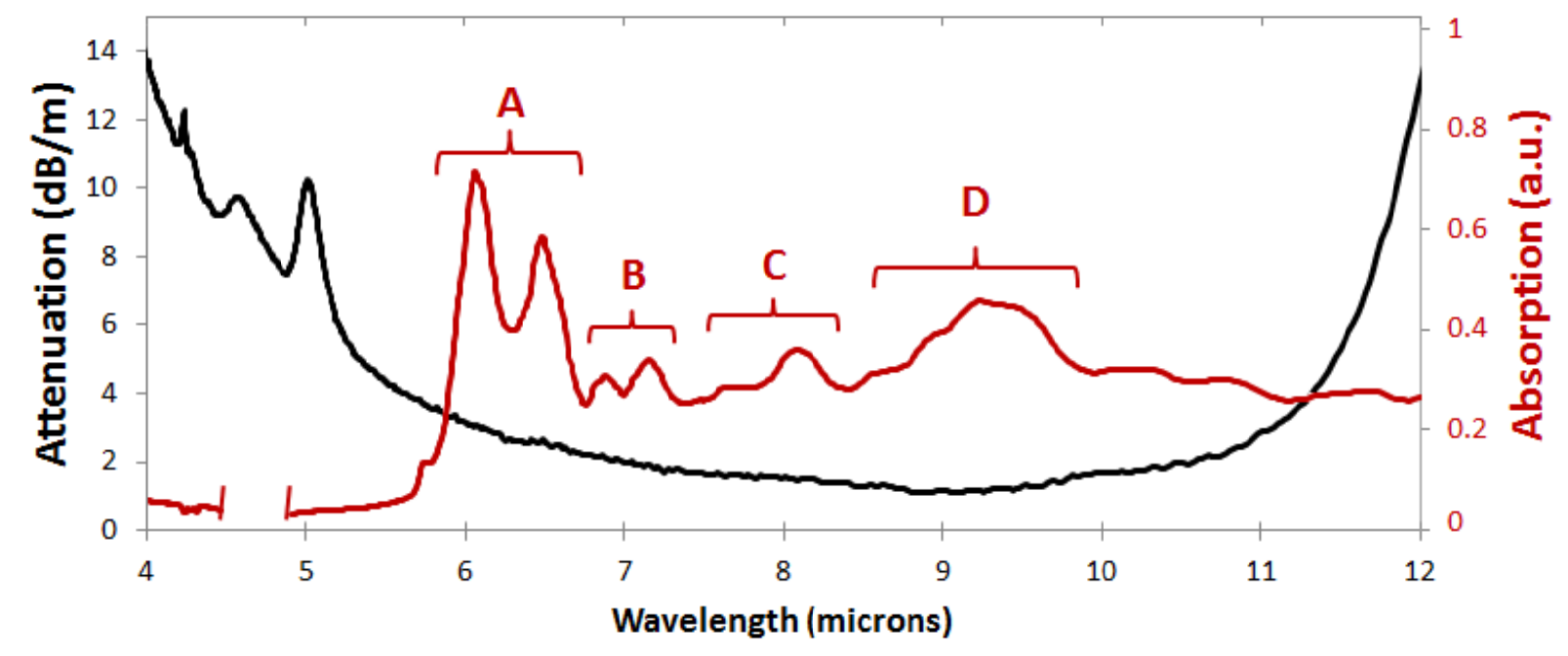

Fig 9: Vibrational signature of human lung cells collected using a tapered chalcogenide fiber sensor. The signal of each cell component can be identify including A: Amide I and II; B: fatty acids and amino acids; $C$ : phospholipids and nucleic acids; D: polysaccharides. Bottom: Optical transmission of a $\mathrm{Ge}_{20} \mathrm{As}_{20} \mathrm{Te}_{45} \mathrm{Se}_{15}$ glass fiber.

Strong signals can therefore be collected which enable advanced spectral analysis for selective analysis $[38,43]$. These fibers are now reaching the stage of commercial development for the design of bio-medical sensors but much research is still ongoing to optimize their attenuation and optical window.

\subsection{Toxicity}

Most commercial chalcogenide glass fibers contain significant fraction of arsenic which naturally raise concerns of toxicity for bio-medical applications. But while soluble species of arsenic such as $\mathrm{AsO}_{4}{ }^{3-}$ and $\mathrm{AsO}_{3}{ }^{3-}$ exhibit high toxicity for living organisms, arsenic atoms embedded in a chalcogenide glass are covalently bonded to the amorphous network in a trivalent configuration which make it highly insoluble and results in minimal toxicity. As shown in Fig. 10 chalcogenide glass surfaces show no sign of corrosion upon immersion in aqueous environment for long periods of time. Indeed, As-based fibers have been coated with live cell cultures for in-situ optical monitoring and were shown to produce strong surface attachment of macrophage cells [13]. It was shown that toxicity is insignificant as long as the glass surface is not oxidized [46]. Figure 11 shows a culture of human lung cell forming a confluent layer at the surface of a $\mathrm{Te}_{2} \mathrm{As}_{3} \mathrm{Se}_{5}$ fiber. The viability of the cell was confirmed using a fluorescence assay shown as the bright green color on Fig. 11(b). 

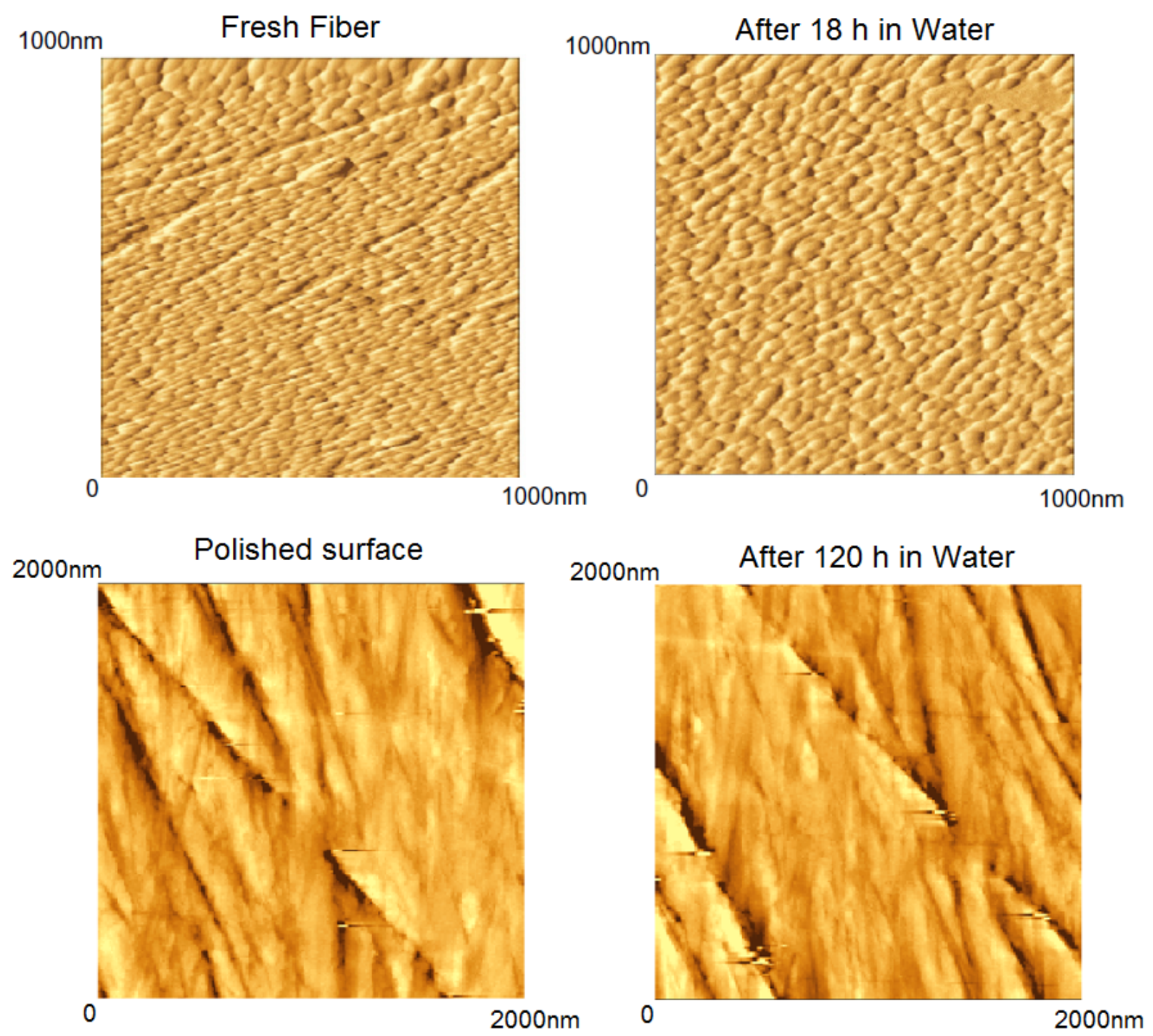

Fig 10: Top: AFM image of a $\mathrm{Te}_{2} \mathrm{As}_{3} \mathrm{Se}_{5}$ glass fiber before and after immersion in water for 18 hours. Bottom: AFM image of a $\mathrm{Ge}_{10} \mathrm{As}_{15} \mathrm{Te}_{75}$ polished glass surface before and after immersion in water for 120 hours. 
(a)

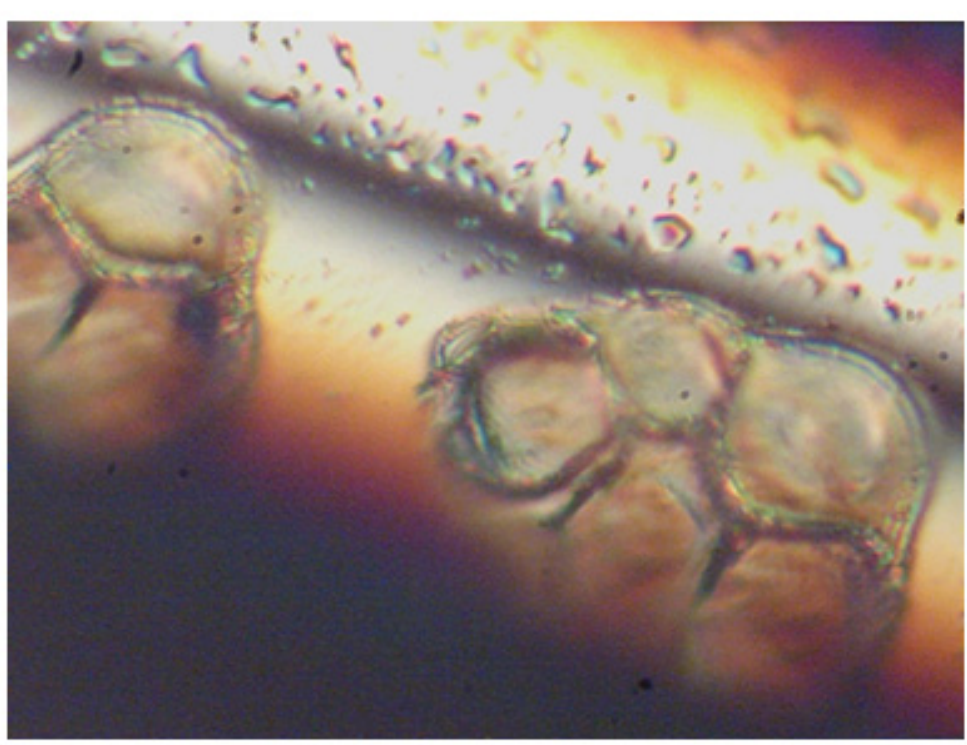

(b)

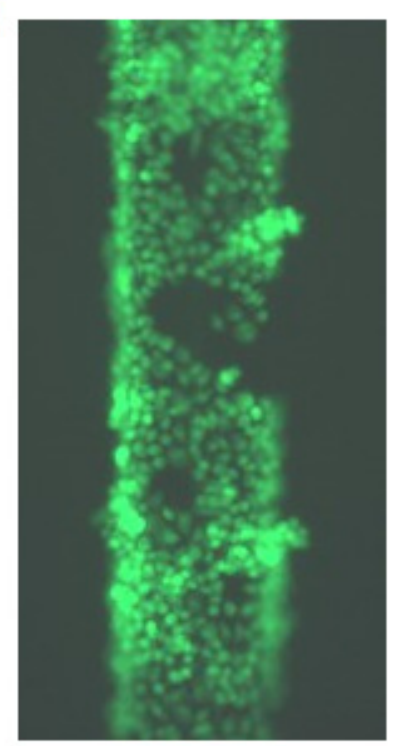

Fig 11: (a): Optical micrograph of human lung cell coated on the surface of a $\mathrm{Te}_{2} \mathrm{As}_{3} \mathrm{Se}_{5}$ glass fiber. (b): Fluorescence photomicrograph of a human lung cell culture forming a confluent layer at the surface of a $\mathrm{Te}_{2} \mathrm{As}_{3} \mathrm{Se}_{5}$ glass fiber. The green colors originate from a fluorescence dye which indicates healthy cells.

\subsection{Mechanical Properties:}

Chalcogenide glasses are known to have weaker mechanical properties than oxide glasses [47]. The corresponding fiber therefore also exhibit modest mechanical resistance with tensile strengths of up to $110 \mathrm{MPa}$ for $\mathrm{Te}_{2} \mathrm{As}_{3} \mathrm{Se}_{5}$ fibers which are shown to decrease with aging due to relaxation and surface degradation [48, 49]. The bending strength is also limited especially for fibers with large diameter. This can be an issue for in-vivo application such as cancer detection. However, the flexibility of a chalcogenide glass fiber can be greatly improved by tapering to thinner diameters as shown in Fig. 12(a) for a $\mathrm{GeSe}_{4}$ fiber. Reduction of the diameter down to $100 \mu \mathrm{m}$ allows bending radius below $1 \mathrm{~cm}$. The taper also extend the evanescent wave and improves the detection limit of the fiber sensor.

In order to improve the robustness of the fiber sensor for ease of handling, looped fiber sensors were produced by hot shaping near the glass transition temperature as shown in Fig. 12(b) [50]. This sensor geometry leads to much improved robustness and opens the way to broader applicability of these fiber devices in the medical field. 
(a)

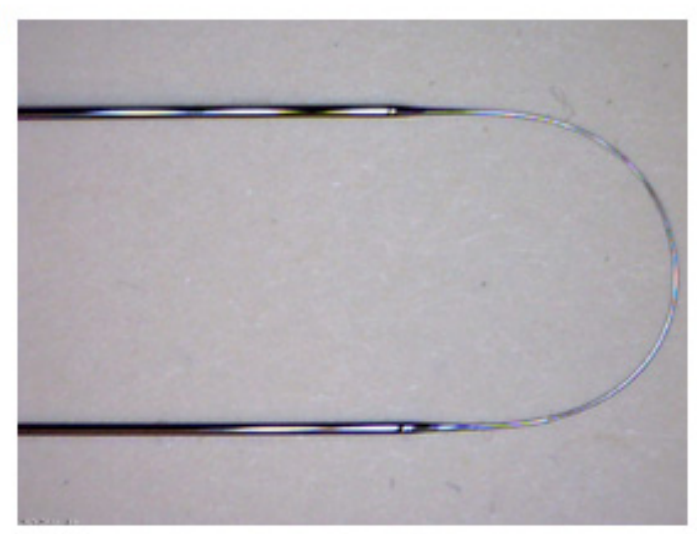

(b)

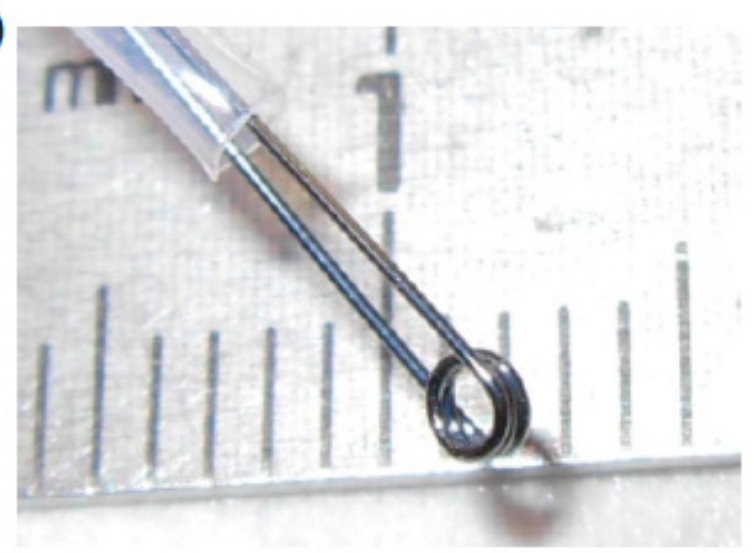

Fig 12: (a): A $300 \mu \mathrm{m}$ diameter GeSe $e_{4}$ optical fiber tapered down to a diameter of $100 \mu \mathrm{m}$ showing a bending radius of less than $1 \mathrm{~cm}$. (b) A looped $\mathrm{Te}_{2} \mathrm{As}_{3} \mathrm{Se}_{5}$ fiber from Ref [50].

\section{Conclusions:}

The design of optical fibers with extended transparency in the infrared region is currently the topic of intensive research. Chalcogenide glasses have proved to be highly versatile materials for that purpose, in part due to the large compositional landscape which enable tailoring the optical and rheological properties of the glass for optimal fiber design. It was shown that telluride glasses are the most promising materials for the production of fibers with long-wave transmission but compositional modifications such as addition of iodine or selenium are required for stabilizing the glass against crystallization while retaining wide optical transparency. Following these principles, low-loss optical fibers with transparency windows optimized for the detection of cell signals can be fabricated. These fibers exhibit low toxicity and can be shaped into robust elements so that they are promising for the development of commercial biomedical sensors.

\section{Acknowledgement:}

This work was supported by the National Science Foundation under Grant Number ECCS1201865 and by the TRIFF Photonic Initiative at the University of Arizona.

\section{REFERENCES}

[1] X. Zhang, B. Bureau, P. Lucas, C. Boussard-Pledel, J. Lucas, Chem.--Eur. J., 14 (2008), pp. 432-442.

[2] Y.M. Guimond, Y. Bellec, Proc. SPIE-Int. Soc. Opt. Eng., 5252 (2004), pp. 103-110. 
[3] X.H. Zhang, Y. Guimond, Y. Bellec, J. Non-Cryst. Solids, 326\&327 (2003), pp. 519-523.

[4] B. Mizaikoff, Anal. Chem., 75 (2003), pp. 258A-267A.

[5] O. Eytan, B.-A. Sela, A. Katzir, Appl. Optics, 39 (2000), pp. 3357-3360.

[6] H. Steiner, M. Jakusch, M. Kraft, M. Karlowatz, T. Baumann, R. Niessner, W. Konz, A. Brandenburg, K. Michel, C. Boussard-Pledel, B. Bureau, J. Lucas, Y. Reichlin, A. Katzir, N. Fleischmann, K. Staubmann, R. Allabashi, J. M. Bayona, B. Mizaikoff, Appl. Spectro., 57 (2003), pp. 607-613.

[7] K. Taga, R. Kellner, U. Kainz, U.B. Sleytr, Anal. Chem., 66 (1994), p. 35.

[8] M. Karlowatz, M. Kraft, B. Mizaikoff, Anal. Chem., 76 (2004), p. 2643.

[9] G. Delaizir, P. Lucas, X. H. Zhang, H. L. Ma, B. Bureau, J. Lucas, J. Am. Cer. Soc., 90 (2007), p. 2073.

[10] D. Naumann, Nature, 351 (1991), p. 81.

[11] M. Diem, K. Papamarkakis, J. Schubert, B. Bird, M.J. Romeo, M. Miljkovic, Appl. Spectrosc., 63 (2009), pp. 307A-318A.

[12] B. Rigas, S. Morgello, I.S. Goldman, P.T.T. Wong, Proc. Natl. Acad. Sci, 87 (1990), p. 8140.

[13] P. Lucas, D. LeCoq, C. Juncker, J. Collier, D.E. Boesewetter, C. Boussard-Pledel, B. Bureau, M.R. Riley, Appl. Spectrosc., 59 (2005), pp. 1-9.

[14] T. Udelhoven, D. Naumann, J. Schmitt, Appl. Spectrosc., 54 (2000), p. 1471.

[15] Z. Yang, M.K. Fah, K.A. Reynolds, J.D. Sexton, M.R. Riley, M.-L. Anne, B. Bureau, P. Lucas, Opt. Express, 18 (2010), pp. 26754-26759.

[16] T. Uemura, K. Nishida, M. Sakakida, K. Ichinose, S. Shimoda, M. Shichiri, Frontiers Med. Biol. Engng., 9 (1999), p. 137.

[17] B.J. Eggleton, B. Luther-Davies, K. Richardson, Nat. Photonics, 5 (2011), pp. 141-148.

[18] C. X. Yu, A. Ganjoo, H. Jain, C. G. Pantano, J. Irudayaraj, Anal. Chem., 78 (2006), p. 2500.

[19] K. Michel, B. Bureau, C. Boussard-Plédel, T. Jouan, J.L. Adama, K. Staubmann, T. Baumannc, Sens. Actuat. B, 101 (2004), pp. 252-259.

[20] P. Lucas, M.R. Riley, C. Boussard-Pledel, B. Bureau, Anal. Biochem., 351 (2006), pp. 1-10.

[21] J. S. Sanghera, F. H. Kung, V. Q. Nguyen, R. E. Miklos, I.D. Aggarwal, Appl. Optics, 33 (1994), pp. 6315-6322.

[22] M. Katz, A. Katzir, I. Schnitzer, A. Bornstein, Applied Optics, 33 (1994), pp. 5888-5894.

[23] Z. Yang, O. Gulbiten, P. Lucas, T. Luo, S. Jiang, J. Am. Ceram. Soc., 94 (2011), pp. 17611765.

[24] R. Frerichs, J. Opt. Soc. Am., 43 (1953), pp. 1153-1157.

[25] A. Feltz, Amorphous Inorganic Materials and Glasses, VCH, Weinheim, 1993.

[26] J. Sanghera, C. Florea, L. Busse, B. Shaw, F. Miklos, I. Aggarwal, Opt. Express, 18 (2010), pp. 26760-26768.

[27] L. Calvez, H. L. Ma, J. Lucas, X.H. Zhang, Adv. Mat., 19 (2007), p. 129.

[28] Y. Ledemi, B. Bureau, L. Calvez, Le Floch, M.; Roze, C. M.; Lin, X.-H. Zhang, M. Allix, G. Matzen, Y. Messaddeq, J. Phys. Chem. B, 113 (2009), pp. 14574-14580.

[29] I. Inagawa, S. Morimoto, T. Yamashita, I. Shirotani, Jpn. J. Appl. Phys., Part 1, 36 (1997), pp. 2229-2235.

[30] J.S. Sanghera, V.Q. Nguyen, P.C. Pureza, F.H. Kung, R. Miklos, I.D. Aggarwal, J. Lightwave Technol., 12 (1994), pp. 737-741.

[31] P. Lucas, C. Boussard-Pledel, A. Wilhelm , S. Danto, X.-H. Zhang, P. Houizot , S. Maurugeon, C. Conseil , B. Bureau, Fibers, 1 (2013), pp. 110-118. 
[32] C. Conseil, J.-C. Bastien, C. Boussard-Pledel, X.-H. Zhang, P. Lucas, S. Dai, J. Lucas, B. Bureau, Opt. Mater. Express, 2 (2012), pp. 1470-1477.

[33] P. Lucas, Z. Yang, M.K. Fah, T. Luo, S. Jiang, C. Boussard-Pledel, M.-L. Anne, B. Bureau, Opt. Mater. Express, 3 (2013), pp. 1049-1058.

[34] Z. Yang, T. Luo, S. Jiang, J. Geng, P. Lucas, Opt. Lett., 35 (2010), pp. 3360-3362.

[35] A.A. Wilhelm, C. Boussard-Pledel, Q. Coulombier, J. Lucas, B. Bureau, P. Lucas, Adv. Mater., 19 (2007), pp. 3796-3800.

[36] Z. Yang, A.A. Wilhelm, P. Lucas, J. Am. Ceram. Soc., 93 (2010), pp. 1941-1944.

[37] P. Lucas, A.A. Wilhelm, M. Videa, C. Boussard-Pledel, B. Bureau, Corros. Sci., 50 (2008), pp. 2047-2052.

[38] P. Lucas, B. Bureau, Advanced infrared glasses for biochemical sensing, in: C.M.P.a.Y.J. Chabal (Ed.) Biointerface Characterization By Advanced IR Spectroscopy, Elsevier 2011, pp. 217-243.

[39] M. Diem, S. Boydston-White, L. Chiriboga, Appl. Spectrosc., 53 (1999), pp. 148A-161A.

[40] D. Naumann, Infrared spectroscopy in microbiology, in: R.A. Meyers (Ed.) Encyclopedia of analytical chemistry, John Wiley \& Sons Ltd, Chichester, 2000, pp. 102.

[41] M. Diem, N. Laver, K. Bedrossian, J. Schubert, K. Papamarkakis, B. Bird, M. Miljkovic, Detection of viral infection in epithelial cells by infrared spectral cytopathology, Handbook of Biophotonics, Wiley-VCH Verlag GmbH \& Co. KGaA2011, pp. 251-258.

[42] S. Hocde, O. Loreal, O. Sire, C. Boussard-Pledel, B. Bureau, B. Turlin, J. Keirsse, P. Leroyer, J. Lucas, J. Biomed. Opt., 9 (2004), pp. 404-407.

[43] M. L. Anne, C. Le Lan, V. Monbet, C. Boussard-Pledel, M. Ropert, O. Sire, M. Pouchard, C. Jard, J. Lucas, J. L. Adam, P. Brissot, B. Bureau, O. Loreal, J. Biomed. Opt., 14 (2009), p. 054033.

[44] J. Keirsse, E. Lahaye, A. Bouter, V. Dupont, C. Boussard-Pledel, B. Bureau, J.-L. Adam, V. Monbet, O. Sire, Appl. Spectrosc., 60 (2006), pp. 584-591.

[45] E. Lepine, Z. Yang, Y. Gueguen, J. Troles, X.-H. Zhang, B. Bureau, C. Boussard-Pledel, J.C. Sangleboeuf, P. Lucas, J. Opt. Soc. Am. B, 27 (2010), pp. 966-971.

[46] A.A. Wilhelm, P. Lucas, D.L. DeRosa, M.R. Riley, J. Mater. Res., 22 (2007), pp. 10981104.

[47] J. P Guin, T. Rouxel, J. C. Sangleboeuf, I. Melscoet, J. Lucas, J. Am. Ceram. Soc., 85 (2002), p. 1545.

[48] G. Yang, H. Chen, C. Boussard-Pledel, J.-C. Sangleboeuf, B. Bureau, Ceram. Int., 41 (2015), pp. 4487-4491.

[49] G. Delaizir, J.-C. Sangleboeuf, E.A. King, Y. Gueguen, X.-H. Zhang, C. Boussard-Pledel, B. Bureau, P. Lucas, J. Phys. D: Appl. Phys., 42 (2009), pp. 095405/095401-095406.

[50] P. Houizot, M.-L. Anne, C. Boussard-Pledel, O. Loreal, H. Tariel, J. Lucas, B. Bureau, Sensors, 14 (2014), pp. 17905-17914. 